\title{
Evaluación de la eficacia de algunos fármacos para el tratamiento de la hepatozoonosis canina
}

\section{Evaluation of the effectiveness of some drugs for the treatment of canine hepatozoonosis}

\author{
Guendulain $\mathbf{C}^{1}$, González $\mathbf{G}^{1}$, Babini S1, Caffaratti $\mathbf{M}^{1}$, \\ González $\mathbf{P}^{1}$, Bessone $\mathbf{A}^{1}$, Soler $\mathbf{E}^{2}$, Tissera $\mathbf{M C}^{1}$ \\ ${ }^{1}$ Departamento de Clínica Animal, Facultad de Agronomía y Veterinaria; ${ }^{2}$ Departamento de Matemática y \\ Estadística, Facultad de Ciencias Económicas. Universidad Nacional de Río Cuarto. \\ ${ }^{*}$ Correo electrónico del autor: cguendulain@ayv.unrc.edu.ar
}

\begin{abstract}
Resumen: La hepatozoonosis canina es una enfermedad parasitaria, adquirida por la ingestión de garrapatas infectadas con protozoarios del género Hepatozoon $(H)$. Hepatozoon canis y Hepatozoon americanum son las dos especies que pueden infectar al perro. La presentación clínica de la infección con Hepatozoon canis es muy variable, pudiendo ser asintomática o manifestarse con signos de enfermedad leves a severos. Los exámenes hematológicos revelan ligera anemia no regenerativa, marcada leucocitosis neutrofílica con desvío a la izquierda y monocitosis en los casos de hepatozoonosis clínica. El diagnóstico se realiza mediante la visualización mediante el microscopio óptico de los gamontes en neutrófilos y monocitos en frotis de sangre coloreados. No existe, hasta el momento, un tratamiento eficaz para esta parasitosis. El fármaco más utilizado es el dipropionato de imidocarb, aunque con resultados variables. El objetivo de este trabajo fue evaluar tres opciones terapéuticas para la erradicación de Hepatozoon spp. de la sangre de perros infectados. Se utilizaron 18 perros parasitados naturalmente y se evaluaron tres fármacos: dipropionato de imidocarb, toltrazuril y espiramicina. En todos los perros tratados disminuyó el promedio de infección, presentando similares valores medios de leucocitos infectados. El análisis estadístico arrojó una diferencia significativa solo en el tratamiento de la parasitosis con dipropionato de imidocarb. En virtud de los resultados obtenidos con los tratamientos llevados a cabo con los diferentes fármacos, se concluye que ninguno es totalmente efectivo para la desaparición del parásito de la sangre, siendo el dipropionato de imidocarb el de mejor comportamiento.
\end{abstract}

Palabras clave: Hepatozoon, perros, tratamiento

\begin{abstract}
Canine hepatozoonosis is a parasitic disease, acquired by ingestion of ticks infected with protozoa of the genus Hepatozoon $(\mathrm{H})$. Hepatozoon canis and Hepatozoon americanum are the two species that can infect dogs. The clinical presentation of Hepatozoon canis infection is highly variable and may be asymptomatic or show signs of mild to severe disease. In clinical hepatozoonosis, haematological tests reveal slight non regenerative anemia, marked neutrophilic leukocytosis with left shift and monocytosis. Diagnosis is made by the visualization of gamonts in neutrophils and monocytes in stained blood smears using an optical microscope. To date, there is no effective treatment for hepatozoonsis; imidocarb propionate is the most commonly used drug, although results are variable. The aim of this study was to evaluate three therapeutic options for the eradication of Hepatozoon spp. from blood of infected dogs. Three drugs (imidocarb dipropionate, toltrazuril and spiramycin) were evaluated in 18 naturally infected dogs. In all treated dogs the infection averages decreased, showing similar mean values of infected leukocytes. The statistical analysis showed a significant difference only in the treatment of this parasitosis with imidocarb dipropionate. It is concluded that none of the drugs are fully effective in removing the parasite from the blood, but imidocarb dipropionate showed the best results.
\end{abstract}

Key words: Hepatozoon, dog, treatment 


\section{Introducción}

La hepatozoonosis canina es una enfermedad parasitaria, adquirida por la ingestión de garrapatas infectadas con protozoarios del género Hepatozoon $(H)$. El perro puede infectarse por dos especies de este género: Hepatozoon canis, transmitido por Rhipicephalus sanguineus, la garrapata marrón del perro (Baneth et al., 2001), y Hepatozoon americanum (VincentJohnson et al., 1997), transmitido por Amblyomma maculatum, la garrapata de la Costa del Golfo (Ewing et al., 2002; Mathew et al., 2000). En América del Sur la especie reconocida hasta el momento es Hepatozoon canis. En Argentina, la enfermedad se describió por primera vez en el año 1999 (Silva et al., 1999), y recién en el año 2007 se realizó la primera caracterización molecular, confirmando que la especie estudiada era Hepatozoon canis (Eiras et al., 2007).

El perro se parasita al ingerir una garrapata con esporozoitos, los cuales colonizan distintos órganos: hígado, bazo y linfonódulos (Hepatozoon canis). La infección de la garrapata vector se produce cuando ingiere sangre de un perro con leucocitos parasitados con gamontes de Hepatozoon spp. (Baneth et al., 2007).

La infección puede ser asintomática, y el parásito encontrarse accidentalmente en animales sanos o que padecen otra enfermedad, o puede manifestarse como una enfermedad severa, potencialmente mortal (Baneth \& Weigler, 1997; Baneth et al., 2007). Los signos clínicos observados con mayor frecuencia son mucosas pálidas, emaciación y fiebre intermitente (Gavazza et al., 2003). La deficiencia del sistema inmune en animales jóvenes, la presencia de otros agentes infecciosos; o los tratamientos con agentes inmunosupresores tienen un alto impacto en la persistencia o en la presentación de una nueva infección (Baneth et al., 2001; Elias \& Homans, 1988; Mylonakis et al., 2005).

No existe hasta el momento un protocolo de tratamiento totalmente efectivo para la infección con Hepatozoon canis, por lo que cobra importancia la prevención de la infección basada en el control efectivo de las garrapatas sobre el perro y el ambiente (Baneth et al., 2007).

El fármaco más utilizado es el dipropionato de imidocarb (5-6 mg/kg), por vías SC o IM, cada 14 días, hasta la desaparición de los gamontes en sangre, aunque los resultados son variables (Baneth \& Weigler 1997; Pasa et al., 2011).Usualmente, una o dos aplicaciones son suficientes, pero en infecciones severas puede necesitarse un tratamiento de hasta ocho semanas (Macintire et al., 2001). La doxiciclina (10 mg/kg), administrada por vía oral durante 21 días, suele utilizarse en asociación con el dipropionato de imidocarb, debido a las posibles coinfecciones con otros protozoarios transmitidos por la garrapata (Ba- neth et al., 2003). Otra alternativa de tratamiento es el toltrazuril, aunque la eficacia también es variable (Krampitz \& Haberkorn 1988; Macintire, 2001).

A pesar de que el dipropionato de imidocarb es el fármaco recomendado para la hepatozoonosis, su utilización no garantiza la cura parasitológica. En un ensayo se logró la eliminación de los gamontes de la sangre de perros en el $98 \%$ de los casos dentro de las 24 horas posteriores a la administración de una única dosis. Sin embargo, en muchos casos se produjo la reaparición de la parasitemia pocas semanas después (Ogunkoya, 1981). En otro estudio, en el que se realizó el seguimiento de tres perros tratados durante ocho meses, se determinó, mediante la observación de frotis sanguíneos y métodos moleculares, que la droga no fue efectiva para la eliminación de los gamontes de la sangre. Si bien al finalizar el tratamiento no se observaron parásitos en las muestras de sangre mediante microscopía óptica, sí se detectaron mediante PCR (Sasanelli et al., 2010).

También se ha estudiado el dipropionato de imidocarb en asociación con otras drogas con pobres resultados. Administrado en forma conjunta con tetraciclinas (Elias \& Homans, 1988; Parra \& Arraga de Alvarado, 1996) y con doxiciclina (Baneth et al., 1995).

Mediante el uso de toltrazuril también se han demostrado resultados dispares en el tratamiento de las infecciones por Hepatozoon. Usado en perros infectados (10 mg/kg), por vía oral, durante seis días, ocurrió la remisión de los signos clínicos y la normalización de los parámetros hematológicos, pero no se eliminó la infección (Beaufils et al., 1996). Pérez Tort et al. (2007) comunicaron la recuperación clínica y la desaparición de los gamontes de la sangre de doce perros infectados con una dosis diaria de $14 \mathrm{mg} / \mathrm{kg}$, por vía oral, durante siete días.

Del mismo modo que ocurrió con el dipropionato de imidocarb, el toltrazuril se estudió en asociación con otras drogas. Voyvoda et al. (2004) utilizaron con éxito toltrazuril con trimetoprim-sulfametoxazol en un caso clínico, aunque el tratamiento con trimetoprimsulfametoxazol se realizó durante 25 días y debió repetirse el toltrazuril, ya que en la primera administración no se logró la eliminación completa del parásito.

Por otra parte, Pasa et al. (2011) realizaron un estudio usando una asociación de dipropionato de imidocarb y toltrazuril en seis perros, concluyendo que la incorporación de toltrazuril no produjo un beneficio adicional con respecto a la terapia con dipropionato de imidocarb como única droga. En un estudio realizado con tres grupos de once perros cada uno, se evaluó la eficacia del dipropionato de imidocarb por un lado, y del toltrazuril/emodepside en asociación con clindamicina por otro. Los resultados indicaron que ninguna de los dos tratamientos lograron la eliminación del 
parásito: si bien se produjo disminución del porcentaje de animales infectados, no se logró una completa cura parasitológica (De Tommasi et al., 2014).

A pesar de que con los tratamientos implementados los resultados son dispares, un alto porcentaje de perros que posee baja parasitemia se cura, sobre todo si no existen otras enfermedades concomitantes. En ocasiones, luego del tratamiento se logra la mejoría clínica del paciente, aunque no se logra erradicar el parásito de la sangre. Se recomienda realizar el tratamiento en todos los perros infectados, incluidos aquellos con signos leves, debido a que la parasitemia puede incrementarse y desencadenar una enfermedad severa (Baneth \& Weigler, 1997). Se ha informado un intervalo de 28-42 días entre el comienzo del tratamiento y la desaparición de los gamontes de la sangre, probablemente porque desaparecen de la sangre como consecuencia de la muerte de los estadios tisulares de Hepatozoon canis (Elias \& Homans 1988). Por otra parte, se sugirió que el tratamiento no es efectivo contra los parásitos que se encuentran en los tejidos, por lo cual pueden ocurrir recidivas (Vincent-Johnson et al., 1997).

En ocasiones, durante el tratamiento el porcentaje de neutrófilos parasitados aumenta, a pesar deproducirse, en algunos casos, la mejoría clínica del paciente; en otros casos, en cambio, se produce un desmejoramiento clínico coincidente con el aumento de la parasitemia. No se conoce bien el mecanismo por el cual se produce este efecto (Beaufils, 1996; Sasanelli et al., 2010; Voyvoda et al., 2004).

La espiramicina es un antibiótico macrólido, bacteriostático, con un potencial particular contra organismos intracelulares ya que se concentra en el interior de las bacterias y de los macrófagos, por lo que presenta un largo efecto post antibiótico (Giguere et al., 2013; Greene, 2008).

El objetivo de este estudio fue evaluar tres fármacos: dipropionato de imidocarb, toltrazuril y espiramicina para la erradicación del Hepatozoon spp. de la sangre de perros infectados.

\section{Materiales y métodos Animales en estudio}

Se estudiaron 18 perros infectados naturalmente, entre 6 meses y 13 años de edad, de razas puras y mestizas. Algunos se encontraban en el Centro de Reinserción de Caninos dependiente de la Municipalidad de Río Cuarto y otros eran pacientes que acudieron al Hospital de Clínica Animal (Facultad de Agronomía y Veterinaria -FAV- de la Universidad Nacional de Río Cuarto) con diferentes motivos de consulta.

\section{Toma de muestras y procesamiento}

Se extrajeron $5 \mathrm{ml}$ de sangre de la vena cefálica antebraquial y se colectaron en tubos con anticoagulante (EDTA). Estas muestras fueron procesadas en el Laboratorio de Análisis Clínicos de la FAV, donde se realizaron los frotis sanguíneos coloreados con May Grünwald-Giemsa. El diagnóstico se realizó por la observación de los gamontes en los leucocitos mediante el microscopio óptico. Se determinó el porcentaje de leucocitos parasitados (parasitemia relativa) realizando el recuento de los mismos en 100 campos microscópicos con un aumento final de 1000X. Se registraron los datos del paciente y el porcentaje de leucocitos infectados en una ficha elaborada para tal fin. La parasitemia se consideró escasa si se observaban menos del $5 \%$ de los leucocitos parasitados (Baneth et al., 2003), moderada con entre 5-20\% leucocitos parasitados y abundante con $>20 \%$.

\section{Fármacos utilizados}

Se utilizaron dipropionato de imidocarb $(5 \mathrm{mg} /$ $\mathrm{kg}$ vía SC, cada 14 días), toltrazuril (14 mg/kg vía oral, cada $24 \mathrm{~h}$, durante 7 días) y espiramicina $(23 \mathrm{mg} / \mathrm{kg}$ vía oral, cada $24 \mathrm{~h}$, durante 10 días). Las dosis y la frecuencia de administración de las drogas utilizadas fueron las indicadas por la bibliografía (Giguere et al., 2013; Greene, 2008; Macintire, 1999).

\section{Grupos experimentales}

Para la administración de las drogas se confeccionaron al azar tres grupos de seis perros cada uno, infectados naturalmente con Hepatozoon spp. La mayoría de los perros no presentaba signos clínicos específicos de hepatozoonosis ni otras entidades concomitantes. A todos los animales se les realizó hemograma y frotis sanguíneo previos al tratamiento (día 0). Una semana antes de comenzar los tratamientos se les colocó un antiparasitario externo a base de imidacloprid, permetrina y butóxido de piperonilo spoton, para realizar el estudio en ausencia de garrapatas en los pacientes.

-Grupo 1: recibió tratamiento con dipropionato de imidocarb. A los 14 días del tratamiento se les realizó un hemograma como control para evaluar la presencia de los gamontes en sangre. A los perros que seguían infectados se les administró una segunda dosis. Se realizaron hasta cuatro aplicaciones en los casos necesarios.

-Grupo 2: recibió tratamiento con toltrazuril.

-Grupo 3: recibió tratamiento con espiramicina.

\section{Seguimiento}

A los perros estudiados se les realizó hemograma de control y frotis de sangre al finalizar el mismo y 
a los 30 días después de comenzado. Los resultados negativos se reconfirmaron con la observación de la capa leucocitaria.

\section{Análisis estadístico}

Para evaluar el resultado de los tratamientos de manera individual se utilizaron pruebas no paramétricas de Wilcoxon para muestras apareadas. El porcentaje de leucocitos infectados fue la variable estudiada para cada una de las tres drogas probadas. Para la confección de la matriz de datos y el análisis estadístico se utilizó el software SPSS.

\section{Resultados}

El resultado de las pruebas de Wilcoxon arrojó una diferencia significativa solo en el tratamiento de la parasitosis con dipropionato de imidocarb $(p=0,002)$. Los valores medios de leucocitos parasitados antes y después de los tratamientos pueden observarse en la Tabla 1.

Tabla 1. Valores medios del porcentaje de leucocitos infectados con Hepatozoon spp.de los grupos de perros con tratamientos.

\begin{tabular}{lcc}
\hline GRUPO & $\begin{array}{c}\text { ANTES } \\
\text { (Valores } \\
\text { medios) }\end{array}$ & $\begin{array}{c}\text { DESPUÉS } \\
\text { (Valores } \\
\text { medios) }\end{array}$ \\
\hline dipropionato de imidocarb & $11,17 \%$ & $0,50 \%$ \\
toltrazuril & $7,50 \%$ & $3,50 \%$ \\
espiramicina & $3,50 \%$ & $2,67 \%$ \\
\hline
\end{tabular}

En el tratamiento con dipropionato de imidocarb se observó que, en cuatro de los seis casos en los que la parasitemia era escasa $(<5 \%$, casos $2,3,4$ y 6$)$, con una única dosis dejaron de observarse leucocitos parasitados. En el caso en el que la parasitemia era moderada ( $5-20 \%$, caso 1 ), también se logró un $0 \%$ de leucocitos parasitados, pero luego de cuatro dosis. En el caso en el que la parasitemia era abundante $(>20 \%$, caso 5), se requirieron cuatro dosis para disminuir el valor a $3 \%$, pero no se eliminó completamente la parasitemia (Tabla 2).

Con el tratamiento con toltrazuril se observó que, en uno de los dos casos en los que la parasitemia era escasa (3\%), se llegó a $0 \%$ de leucocitos parasitados pero, en el otro (2\%), dicho porcentaje aumentó levemente (3\%). En dos de los casos en que la parasitemia era moderada ( $6 \%$ y $18 \%$ ) disminuyeron a $0 \%$ los leucocitos parasitados; en otro (8\%) disminuyó a $4 \%$ y en otro caso aumentó de $8 \%$ a $14 \%$ (Tabla 3 ).
Tabla 2. Porcentaje de leucocitos parasitados antes y después del tratamiento con dipropionato de imidocarb y cantidad de dosis que se administraron para llegar a $0 \%$.

\begin{tabular}{cccc}
\hline $\begin{array}{c}\text { Perro } \\
\mathbf{N}^{\circ}\end{array}$ & $\begin{array}{c}\text { ANTES } \\
\text { (\% de leuco- } \\
\text { citos parasita- } \\
\text { dos) }\end{array}$ & $\begin{array}{c}\text { DESPUÉS } \\
\text { (\% de leuco- } \\
\text { citos parasita- } \\
\text { dos) }\end{array}$ & $\begin{array}{c}\text { cantidad } \\
\text { de dosis }\end{array}$ \\
\hline $\mathbf{1}$ & $13 \%$ & $0 \%$ & 4 \\
$\mathbf{2}$ & $3 \%$ & $0 \%$ & 1 \\
$\mathbf{3}$ & $1 \%$ & $0 \%$ & 1 \\
$\mathbf{4}$ & $3 \%$ & $0 \%$ & 1 \\
$\mathbf{5}$ & $46 \%$ & $3 \%$ & 4 \\
$\mathbf{6}$ & $1 \%$ & $0 \%$ & 1 \\
\hline
\end{tabular}

Tabla 3. Porcentaje de leucocitos parasitados antes y después del tratamiento con toltrazuril.

\begin{tabular}{ccc}
\hline $\begin{array}{c}\text { Perro } \\
\mathbf{N}^{\circ}\end{array}$ & $\begin{array}{c}\text { ANTES } \\
\text { (\% de leucocitos } \\
\text { parasitados) }\end{array}$ & $\begin{array}{c}\text { DESPUÉS } \\
\text { (\% de leucocitos para- } \\
\text { sitados) }\end{array}$ \\
\hline $\mathbf{1}$ & $18 \%$ & $0 \%$ \\
$\mathbf{2}$ & $3 \%$ & $0 \%$ \\
$\mathbf{3}$ & $8 \%$ & $14 \%{ }^{* *}$ \\
$\mathbf{4}$ & $8 \%$ & $4 \%$ \\
$\mathbf{5}$ & $6 \%$ & $0 \%$ \\
$\mathbf{6}$ & $2 \%$ & $3 \%$ ** \\
\hline
\end{tabular}

${ }^{*}$ Casos en los que se registró un aumento en el porcentaje de leucocitos parasitados luego del tratamiento

Luego del tratamiento con espiramicina se observó que, en los cuatro casos en que la parasitemia era escasa, el porcentaje de leucocitos parasitados disminuyó a $0 \%$. Con respecto a los dos casos en que la parasitemia era moderada, en uno disminuyó el porcentaje $(2 \%)$ y en el otro hubo un aumento del valor (14\%) (Tabla 4).

En todos los perros tratados disminuyeron los promedios de infección, presentando valores medios de leucocitos infectados similares (Tabla 1).

\section{Discusión y conclusiones}

En este estudio se utilizaron el dipropionato de imidocarb, por ser considerado de elección por la comunidad científica para el tratamiento de esta parasitosis, y el toltrazuril porque es otro de los fármacos más utilizados, por los profesionales de nuestro país, debido a que existe una formulación disponible para su uso en pequeños animales indicada para esta parasitosis. La espiramicina se eligió por poseer una eficacia potencial particular contra organismos intracelulares, 
Tabla 4. Porcentaje de leucocitos parasitados antes y después del tratamiento con espiramicina

\begin{tabular}{lcc}
\hline $\begin{array}{l}\text { Perro } \\
\mathbf{N}^{\circ}\end{array}$ & $\begin{array}{c}\text { ANTES } \\
\text { (\% de leucocitos para- } \\
\text { sitados) }\end{array}$ & $\begin{array}{c}\text { DESPUÉS } \\
\text { (\% de leucocitos } \\
\text { parasitados) }\end{array}$ \\
\hline $\mathbf{1}$ & $7 \%$ & $2 \%$ \\
$\mathbf{2}$ & $1 \%$ & $0 \%$ \\
$\mathbf{3}$ & $3 \%$ & $0 \%$ \\
$\mathbf{4}$ & $6 \%$ & $14 \%$ \\
$\mathbf{5}$ & $2 \%$ & $0 \%$ \\
$\mathbf{6}$ & $2 \%$ & $0 \%$ \\
\hline
\end{tabular}

**Caso en el que se registró un aumento del porcentaje de leucocitos parasitados luego del tratamiento

permanecer por más tiempo que otros fármacos de su grupo dentro de los leucocitos, estar disponible en el mercado, ser de fácil administración y no presentar efectos adversos (Giguere et al., 2013).

Mediante los resultados de este trabajo se puso en evidencia que, aunque en general se constató una reducción en la cantidad de leucocitos parasitados, ninguna de las tres opciones fue totalmente eficaz para lograr la eliminación del parásito de la sangre de los perros infectados. Algunos perros respondieron bien al tratamiento y desaparecieron los leucocitos parasitados, pero otros no mostraron una respuesta del todo favorable, ya que si bien la carga parasitaria disminuyó, no se logró la eliminación total de la parasitemia; hubo, inclusive, unos pocos casos en los cuales la parasitemia aumentó.

El dipropionato de imidocarb resultó la mejor opción terapéutica, dado que, de 6 casos, en 5 se eliminó el parásito de la sangre y en el restante (el caso de parasitosis abundante, $46 \%$ ) se redujo el porcentaje a $3 \%$. Con respecto a los animales tratados con toltrazuril y espiramicina, solo se logró la eliminación del parásito en la sangre de 3 y 4 pacientes, respectivamente. A su vez, luego del tratamiento con toltrazuril y espiramicina se observó aumento de la parasitemia en un caso de cada uno. Esto coincide con datos de la bibliografía en que se informan resultados inconstantes con la utilización de dipropionato de imidocarb y toltrazuril (Baneth et al., 1995; Beaufils et al., 1996; De Tommasi et al., 2014; Ogunkoya et al., 1981; Parra \& Arraga-Alvarado 1996; Pasa et al., 2011; Sasanelli et al., 2010).

En cuanto al tercer fármaco, la espiramicina, a pesar de que los resultados obtenidos en este estudio no fueron satisfactorios, queda abierta la posibilidad de nuevas investigaciones con una muestra más representativa con una mayor cantidad de animales.

Aunque el dipropionato de imidocarb es, por el momento, el fármaco considerado de elección para el tratamiento de la hepatozoonosis canina, existen estudios que muestran que no es completamente eficaz para lograr la cura parasitológica de los perros infectados (Ogunkoya 1981; Sasanelli et al., 2010). En coincidencia con esto, los resultados obtenidos en esta investigación con este fármaco no fueron totalmente efectivos, pero es de destacar que en casi todos los animales se logró eliminar la parasitemia. La mayoría de los perros que fueron tratados con dipropionato de imidocarb no presentaban signos clínicos ni entidades patológicas concomitantes, por lo cual es factible que su sistema inmune hubiera contribuido con la cura parasitológica (Baneth \& Weigler 1997, Baneth et al., 2001, Baneth et al., 2007). Uno de los perros, con alto valor de parasitemia, sí presentaba signos clínicos compatibles con esta parasitosis. Con el tratamiento, si bien se necesitaron cuatro dosis, se produjo una reducción importante de la parasitemia ( $46 \%$ a $3 \%)$.

Por su parte, el toltrazuril ha mostrado resultados dispares en el tratamiento de las infecciones por Hepatozoon en coincidencia con Beaufils et al. (1996) y Pérez Tort et al. (2007). De manera similar a lo que ocurrió con el grupo de perros tratados con dipropionato de imidocarb, la mayoría de los perros tratados con toltrazuril no presentaban signos clínicos ni entidades patológicas concomitantes. Uno solo de las perros tenía signos clínicos severos, como paraparesia no ambulatoria y dolor generalizado, compatibles con hepatozoonosis y, a pesar de ésto, no se encontraron gamontes en los leucocitos. Luego de una semana, al realizar nuevamente la observación del frotis sanguíneo, se encontró un $6 \%$ de leucocitos parasitados. Se comenzó el tratamiento con toltrazuril y desaparecieron los signos clínicos en forma rápida, en aproximadamente 24 horas, pero aumentó el porcentaje de parasitemia. Este aumento fue reportado por otros autores tanto con el uso de toltrazuril como de dipropionato de imidocarb (Baneth et al., 1995; Sasanelli et al., 2010; Voyvoda et al., 2004). Baneth et al. (1995) evidenciaron este efecto usando dipropionato de imidocarb con doxiciclina, aunque la condición clínica del perro desmejoró y volvió a recuperarse al disminuir la parasitemia; ésto no sucedió en el caso del presente estudio. Voyvoda et al. (2004) reportaron en un perro con infección severa, un aumento de $21 \%$ a $26 \%$ de neutrófilos parasitados al día 10 de tratamiento con una asociación de toltrazuril y trimetoprim-sulfametoxazol, aunque con una mejoría de la condición clínica, que se evidenció dentro de las $72 \mathrm{~h}$ de comenzado el tratamiento. Esta observación fue hecha también por Sasanelli et al. (2010), quienes encontraron que en uno de los perros la parasitemia aumentó abruptamente de $0,67 \%$ a $4 \%$ durante el tratamiento con dipropionato de imidocarb. Se desconoce la causa de este aumento de la parasitemia durante el tratamiento, por lo cual sería un aspecto a 
estudiar en un futuro para lograr un conocimiento más acabado del comportamiento de este parásito en el perro. Baneth et al. (1995) sugieren que este aumento de los neutrófilos parasitados, podría deberse a la dispersión generalizada de los merontes desde los tejidos. También sugieren que el tiempo entre la realización del tratamiento y la desaparición del parásito de la sangre, que se ha reportado como de 28 a 42 días con el uso de dipropionato de imidocarb y tetraciclina (Elías \& Homans, 1988), se podría deber a que se lleva a cabo un largo proceso, en el cual primero se produce la muerte de los merontes en los tejidos, luego la liberación de los merozoítos desde los merontes y seguidamente la disminución de los gamontes en los leucocitos circulantes (Baneth et al., 1995).

Con respecto a la espiramicina, los perros tratados con este fármaco tampoco presentaban signos clínicos compatibles con esta parasitosis ni entidades patológicas concomitantes. En cuanto a los resultados obtenidos con su uso, no fueron los esperados, a pesar de ser un fármaco efectivo contra organismos intracelulares, de lograr una concentración sanguínea persistente y de ser recomendado para el tratamiento de la toxoplasmosis en medicina humana (Couvreur et al., 1988). De todos modos, si bien no fue efectiva en el $100 \%$ de los casos, en la mayoría se logró la negativización en sangre de la parasitosis, por lo que se sugieren futuros ensayos con una mayor cantidad de perros.

El hecho de que los tratamientos no fueran del todo eficaces para lograr la eliminación del parásito de la sangre, podría deberse, como lo sugieren De Tommasi et al. (2014), a que los fármacos utilizados no son totalmente eficaces contra el parásito, o a que no alcanzan el nivel óptimo para ejercer su efecto en los tejidos donde se enquista el Hepatozoon, tales como el bazo y la médula ósea. Dado que algunos perros, aún sin tratamiento, disminuyeron la carga parasitaria, se podría pensar que el parásito tiene una actividad cíclica entre la sangre y los órganos de depósito, que el sistema inmune del huésped juega un rol verdaderamente importante y/o que existe una susceptibilidad individual para la infección.

En virtud de los resultados obtenidos en el presente estudio, con los tratamientos llevados a cabo con los diferentes fármacos, se concluye que ninguno de los tratamientos es totalmente efectivo para la desaparición del parásito de la sangre, siendo el dipropionato de imidocarb el de mejor comportamiento. Se destaca la importancia de realizar futuras investigaciones a fin de profundizar el estudio de estos u otros fármacos para el tratamiento de la hepatozoonosis canina.

\section{Conflicto de intereses}

Los autores declaran que no existe conflicto de intereses, relaciones financieras, personales o de otro tipo con personas u organizaciones que pudieran afectar al presente trabajo.

\section{Bibliografía}

Baneth G, Harmelin A, Presentey BZ. 1995. Hepatozoon canis infection in two dogs. Journal of the American Veteterinary Medical Association. 206(12): 1891-94.

Baneth G, Mathew JS, Shkap V, Macintire DK, Barta JR, Ewing SA. 2003. Canine hepatozoonosis: two disease syndromes caused by separate Hepatozoon spp. Trends in Parasitology. 19(1):27-31.

Baneth G, Samish M, Alekseev Y, Aroch I, Shkap V. 2001. Transmission of Hepatozoon canis to dogs by naturally fed or percutaneously injected Rhipicephalus sanguineus ticks. Journal of Parasitology. (87):606-11.

Baneth G, Samish M, Shkap V. 2007. Life cycle of Hepatozoon canis (Apicomplexa: Adeleorina: Hepatozoidae) in the tick Rhipicephalus sanguineus and domestic dog (Canis familiaris). Journal of Parasitology. (93):283-99.

Baneth G. Hepatozoonosis. Infección por Hepatozoon canis. En: Greene CE. 2008. Enfermedades infecciosas del perro y el gato. $3^{\circ}$ Ed. Vol. 2. Buenos Aires: Ed. Intermédica. pp. 1387-452.

Baneth G, Weigler B. 1997. Retrospective case-control study of hepatozoonosis in dogs in Israel. Journal of Veterinary Internal Medicine. 11(6):365-70.

Beaufils JP, Martin-Granel J, Jumelle PH. 1996. Hépatozoonose chez le chien et chez le renard: épidemiologie, clinique e traitment. Pratique Médicale et Chirurgicale de l'Animal de Compagnie. 33:243-53.

Couvreur J, Desmonts G, Thulliez P. 1988. Prophylaxis of congenital toxoplasmosis. Effects of spiramycin on placental infection. Journal of Antimicrobial Chemotherapy. 22B:193200.

De Tommasi AS, Alessio G, De Caprariis D, Ramos G, Di Paola RAN, Crescenzo G, Dantas-Torres F, Baneth G, Otranto D. 2014. Failure of imidocarb dipropionate and toltrazuril/emodepside plus clindamycin in treating Hepatozoon canis infection. Veterinary Parasitology. 200(3-4):242-5. doi: 10.1016/j.vetpar.2013.12.013

Eiras DF, Basabe J, Scodellaro CF, Banach DB, Matos ML, Krimer A, Baneth G. 2007. First molecular characterization of canine hepatozoonosis in Argentina: evaluation of asymptomatic Hepatozoon canis infection in dogs from Buenos Aires. Veterinary Parasitology. 149(3-4):275-9.

Elias E, Homans PA. 1988. Hepatozoon canis infection in dogs: clinical and hematological findings and treatment. Journal of Small Animal Practice. 29:55-62.

Ewing SA, Mathew JS, Panciera RJ. 2002. Transmission of Hepatozoon americanum (Apicomplexa: Adeleorina) by Ixodides (Acari: Ixodidae). Journal of Medical Entomology. 39(4):631-4.

Gavazza A, Bizzeti M, Papini R. 2003. Observations on dogs found naturally infected with Hepatozoon canis in Italy. Revue de Medecine Veterinaire. 159:565-71.

Giguère S. Macrolides, azalides, and ketolides. En: Giguère S, Prescott JF, Dowling PM, 2013. Antimicrobial Therapy in Veterinary Medicine. $5^{\circ}$ Ed. New Jersey (United States). 
Wiley-Blackwell pp. 211-32.

Krampitz HE, Haberkorn A. 1988. Experimental treatment of Hepatozoon infections with antococcidial agent toltrazuril. Journal of Veterinary Medicine. 35:131-7.

Macintire DK. 1999. Canine hepatozoonosis. 17th Annual Veterinary Medical Forum. American College of Veterinary Internal Medicine. Chicago, USA.

Macintire DK, Vincent-Johnson NA, Kane CW, Lindsay DS, Blagburn BL, Dillonar R. 2001. Treatment of dogs infected with Hepatozoon americanum: 53 Cases (1989-1998). Journal of the American Veteterinary Medical Association. 218(1):77-82.

Mathew JS, van den Bussche RA, Ewing SA, Malayer JR, Latha BR, Panciera RJ. 2000. Phylogenetic relationship of Hepatozoon (Apicomplexa: Adeleorina) based on molecular, morphologic, and life cycle characters. Journal of Parasitology. 86:366-72.

Mylonakis ME, Leontides L, Gonen L, Billinis C, Koutinas AF, Baneth G. 2005. Anti-Hepatozoon canis serum antibodies and gamonts in naturally-occurring canine monocytic ehrlichiosis. Veterinary Parasitology. 129:229-33.

Ogunkoya AB, Adeyanju JB,Aliu Y0. 1981. Experiences with the use of Imizol in treating canine blood parasites in Nigeria. Journal of Small Animal Practice. 22:111-5.

Parra O, Arraga de Alvarado CM. 1996. Hepatozoonosis canina en Venezuela. Hallazgos clínicos y de laboratorio. Revista Científica. 6(2):125-33.

Pasa S, Voyvoda H, Karagenc T, Atasoy A, Gazyagci S. 2011. Failure of combination therapy with imidocarb dipropionate and toltrazuril to clear Hepatozoon canis infection in dogs. Parasitology Research. 109:919-26.

Perez Tort G, Petetta L, Favre ME, Más J, Robles AM. 2007. Primera descripción de un brote de hepatozoonosis en un refugio de perros y su tratamiento mediante una formulación de toltrazuril especialmente preparada para caninos. Veteterinaria Argentina. 24(235).

Sasanelli M,Paradies P, Greco B, Eyal O, Zaza V, Baneth G. 2010. Failure of imidocarb dipropionate to eliminate Hepatozoon canis in naturally infected dogs based on parasitological and molecular evaluation methods. Veterinary Parasitology. 4(171):3-4.

Silva MC, Rodriguez MS, Rosa A, Pereira ME, Marquez AG. 1999. Hepatozoon canis: primer caso en Buenos Aires, Argentina. Revista de Medicina Veterinaria. 6(80):489-92.

Vincent-Johnson NA, Macintire DK, Lindsay DS, Lenz SD, Baneth G, Shkap V, Blagburn BL. 1997. A new Hepatozoon species from dogs: description of the causative agent of canine hepatozoonosis in North America. Journal of Parasitology. (83):1165-72.

Voyvoda H, Pasa S, Uner A. 2004. Clinical Hepatozoon canis infection in a dog in Turkey. Journal of Small Animal Practice. 45:613-17. 